\title{
Estructura del ensamble de peces costeros de los canales y fiordos de la zona central de la Patagonia chilena $\left(48^{\circ}-52^{\circ} \mathrm{S}\right)$
}

\author{
Structure of the coastal fish assemblage from the channels and fjords of \\ central Chilean Patagonia $\left(48^{\circ}-52^{\circ} \mathrm{S}\right)$
}

\author{
Mathias Hüne ${ }^{1,2}$ y Jaime Ojeda ${ }^{1,2,3}$
}

\author{
${ }^{1}$ Instituto de Ecología y Biodiversidad, Casilla 653, Santiago, Chile. mathiashune@gmail.com \\ ${ }^{2}$ Departamento de Ciencias y Recursos Naturales, Universidad de Magallanes, Casilla 113-D, Punta Arenas, Chile \\ ${ }^{3}$ Parque Etnobotánico Omora, Universidad de Magallanes, Puerto Williams, Chile
}

\begin{abstract}
The central coast of Patagonia $\left(48^{\circ}-52^{\circ} \mathrm{S}\right)$ is a particular area to assess the diversity and structure of fish assemblage due to the different oceanographic gradients. In this study, we determined the identity, richness, and diversity indices, and characterized the structure of the coastal fish assemblage by non-parametric analysis of variance using permutations and multidimensional scaling including 6 localities with oceanic influence and 6 localities with continental influence, associated with Western and Eastern sectors of the channel system, respectively. A total of 380 coastal fish specimens, belonging to 17 species from 12 families, were collected. Species richness showed significant differences between the locations analyzed, with greater richness in locations associated with the sectors of East. In turn, there were no significant differences using the Shannon diversity index between groups of stations. Significant differences were found in the composition of assemblages between clusters East-West, identifying 8 species that contributed to the dissimilarity between assemblages by SIMPER test. The Patagonian blenny, Eleginops maclovinus, was the species that most contributed to the dissimilarity, followed by the notothenioid fish Patagonotothen cornucola and silverside Odontesthes regia. The changes observed in species richness and assemblage composition, suggest that the fish from the coastal ecosystem of the channels and fjords of central Patagonia, presents a longitudinal pattern of structuring; regarding the composition of the assemblage between East and West locations, which could be related to environmental variables (e.g., salinity and glacial sedimentation) present in this area.
\end{abstract}

Key words: Ichthyofauna, species richness, spatial distribution, southern Chile

Resumen.- La costa de la zona central de la Patagonia $\left(48^{\circ}-52^{\circ} \mathrm{S}\right)$ representa un área particular para evaluar la diversidad y estructura del ensamble de peces, a causa de los diversos gradientes oceanográficos que exhibe. En este estudio, se determinó la identidad, riqueza, índices de diversidad y se caracterizó la estructura del ensamble de peces costeros mediante análisis de varianza no paramétrico utilizando permutaciones y escalamiento multidimensional. Se incluyó 6 localidades con influencia oceánica y 6 localidades con influencia continental, asociados a sectores Oeste y Este del sistema de canales, respectivamente. Se capturó un total de 380 peces costeros, correspondiente a 12 familias y 17 especies. La riqueza de especies presentó diferencias significativas entre las localidades analizadas, con una mayor riqueza en las localidades asociadas al sector Este. A su vez, no se observaron diferencias significativas mediante el índice de diversidad de Shannon entre los grupos de estaciones. Se observaron diferencias significativas en la composición de los ensambles entre las agrupaciones Este-Oeste, identificándose 8 especies que contribuyeron a la disimilitud entre los ensambles mediante la prueba SIMPER, siendo el róbalo, Eleginops maclovinus, la especie que más contribuyó a la disimilitud, seguido por el pez de piedra, Patagonotothen cornucola, y el pejerrey, Odontesthes regia. Los cambios observados en la riqueza de especies y composición del ensamble, sugieren que los peces en el ecosistema costero de canales y fiordos de la zona central de la Patagonia, presenta un patrón longitudinal de estructuración, en cuanto a la composición del ensamble entre localidades Este y Oeste, que podría estar relacionado a las variables ambientales (e.g., salinidad y sedimentación glacial) presentes en esta zona.

Palabras clave: Ictiofauna, riqueza de especies, distribución espacial, sur de Chile 


\section{INTRODUCCIÓN}

Los canales y fiordos subantárticos de la zona central de la Patagonia, entre el golfo de Penas y el estrecho de Magallanes, conforman un ecosistema complejo por al menos tres razones: i) El sistema de canales y fiordos estuvo cubierto por hielo durante el Ultimo Máximo Glacial, entre 23.000 y 19.000 años atrás (Hulton et al. 2002), el cual produjo varios avances y retrocesos de los campos de hielo patagónicos, modelando la geomorfología de los canales y fiordos durante el cuaternario (Hulton et al. 2002, Silva \& Calvete 2002, Sudgen et al. 2002). Estos cambios ambientales han afectado a la distribución de las especies marinas, debido a la erradicación de ambientes marinos someros y posterior colonización de las áreas libres de hielo (González-Wevar et al. 2012). ii) En el hemisferio sur el sistema de canales y fiordos alberga las áreas de hielo más importantes después de la Antártica, no obstante, su pérdida de masa y el aporte de agua dulce ha sido drástico durante los últimos años (Porter \& Santana 2003, Rignot et al. 2003). De esta forma, los canales y fiordos son influidos por el agua dulce proveniente de ríos y hielos adyacentes que se mezclan con masas de agua de origen subantártico proveniente del Océano Pacífico, originando una masa de agua estuarina (Pickard 1971, Andrade 1991, Pinochet \& Salinas 1996, Sievers \& Silva 2006). iii) Desde una perspectiva longitudinal (Oeste-Este), a partir del Océano Pacífico hacia el continente, la salinidad superficial al igual que la temperatura, disminuyen al interior de los canales, alcanzando valores extremos (salinidad 1) en la cercanía de ventisqueros (Silva \& Calvete 2002). La combinación de estos gradientes sumada a la alta sedimentación glacial en los fiordos interiores (Pickard 1973), determinan un ecosistema particular, formado en ambientes oceanográficos heterogéneos.

En este contexto, la búsqueda de patrones o gradientes que permitan describir la abundancia de especies, distribución y la identificación de ensambles, es crucial para entender la estructura y función de los ecosistemas. Por lo tanto, el análisis de la identidad de las especies, sus medidas de riqueza y diversidad, pueden entregar importante información sobre la estructura, función y estabilidad de las especies que conforman los ensambles. Estas medidas pueden estar asociadas a la dominancia competitiva entre las especies (Kvalseth 1991, Gotelli \& Colwell 2001, Ricotta 2003), así como también a cambios en las condiciones oceanográficas (Worm et al. 2005).

Se ha documentado una menor diversidad de invertebrados bentónicos en los fiordos interiores de la zona central de la Patagonia, siendo atribuido a la alta sedimentación glacial como uno de los principales factores (Thatje \& Mutschke 1999, Ríos et al. 2005, Häussermann 2006). El efecto de los deshielos también afecta significativamente la composición y distribución espacial de los huevos y larvas de ciertos peces epipelágicos y mesopelágicos como la sardina fueguina Sprattus fuegensis (Jenyns, 1842) y el pez hacha Maurolicus parvipinnis (Vaillant, 1888) (Bustos et al. 2011, Landaeta et al. 2011). Sin embargo, trabajos que describan la distribución y abundancia de las especies de peces costeros presentes entre el golfo de Penas al estrecho de Magallanes y frente al Campo de Hielo Patagónico Sur son aún escasos (Sielfeld et al. 2006). Considerando los resultados en invertebrados bentónicos que demuestran una disminución de la riqueza, abundancia y diversidad de especies en un gradiente de Oeste a Este, a medida que disminuye la temperatura, salinidad y aumenta la sedimentación. En el presente trabajo, se busca determinar si la identidad de las especies, la riqueza, la diversidad alfa y la estructura del ensamble de peces costeros, varían en relación a un gradiente longitudinal. Para esto, se analizaron 12 localidades, abarcando 6 sitios con influencia directa del Océano Pacífico y 6 sitios asociados a fiordos con influencia glacial. La identidad, riqueza, abundancia y estructura del ensamble de peces permitirá poner a prueba la predicción sobre (i) una menor riqueza y diversidad de especies en los sitios asociados a fiordos con influencia glacial, (ii) una estructura en términos de identidad y abundancia proporcional diferente entre sitios Este-Oeste, o alternativamente, (iii) los peces costeros no son afectados por las variables oceanográficas, persistiendo una riqueza, diversidad y estructura similar a lo largo del gradiente longitudinal de los canales y fiordos.

\section{Materiales y MÉTODOS}

\section{ÁREA DE ESTUDIO Y MUESTREO}

Se estudió una colección de peces proveniente de 12 sitios de muestreo entre las latitudes $48^{\circ}$ y $51^{\circ} \mathrm{S}$ (Fig. 1). Las muestras fueron recolectadas entre el 28 de enero y 21 de marzo de 2010 y durante el 24 de febrero y 2 de marzo de 2012 (Tabla 1), como parte de las campañas de terreno para muestrear las comunidades bentónicas del sublitoral del Parque Nacional Bernardo O’Higgins y la Reserva Nacional Alacalufes.

Para capturar los peces, se utilizó una red de trasmallo de $60 \mathrm{~m}$ largo, $3 \mathrm{~m}$ alto, 2,5 cm de tamaño de malla interna 
Tabla 1. Fechas y lugares de los muestreos de peces costeros, con detalle de las estaciones Este y Oeste / Dates and locations of the coastal fish sampling, with details of the stations East and West

\begin{tabular}{llcll}
\hline $\begin{array}{l}\text { Estaciones } \\
\text { (categoría longitudinal) }\end{array}$ & \multicolumn{1}{c}{ Fecha } & $\begin{array}{c}\text { Latitud } \\
\left({ }^{\circ} \mathrm{S}\right)\end{array}$ & $\begin{array}{c}\text { Longitud } \\
\left({ }^{\circ} \mathrm{W}\right)\end{array}$ & Área de muestreo \\
\hline 1 (Este) & 28 enero de 2010 & $50^{\circ} 50,7^{\prime}$ & $74^{\circ} 01,6^{\prime}$ & Estero Peel \\
2 (Este) & 29 enero de 2010 & $50^{\circ} 28,3^{\prime}$ & $74^{\circ} 16,9^{\prime}$ & Estero Andrés \\
3 (Este) & 24 febrero de 2010 & $49^{\circ} 08,1^{\prime}$ & $74^{\circ} 26,3^{\prime}$ & Paso del Indio \\
4 (Este) & 02 marzo de 2010 & $49^{\circ} 58,3^{\prime}$ & $74^{\circ} 17,8^{\prime}$ & Seno Penguin \\
5 (Este) & 05 marzo de 2010 & $50^{\circ} 18,8^{\prime}$ & $74^{\circ} 25,5^{\prime}$ & Canal Andrés \\
6 (Este) & 16 marzo de 2010 & $51^{\circ} 02,1^{\prime}$ & $74^{\circ} 09,9^{\prime}$ & Canal Sarmiento \\
7 (Oeste) & 20 marzo de 2010 & $49^{\circ} 10,7^{\prime}$ & $75^{\circ} 23,8^{\prime}$ & Canal Ladrillero \\
8 (Oeste) & 21 marzo de 2010 & $48^{\circ} 54,4^{\prime}$ & $75^{\circ} 06,3^{\prime}$ & Canal Fallos \\
9 (Oeste) & 24 febrero de 2012 & $50^{\circ} 23,2^{\prime}$ & $75^{\circ} 23,9^{\prime}$ & Canal Copihue \\
10 (Oeste) & 26 febrero de 2012 & $50^{\circ} 25,2^{\prime}$ & $75^{\circ} 19,8^{\prime}$ & Seno Contreras \\
11 (Oeste) & 29 febrero de 2012 & $51^{\circ} 13,7^{\prime}$ & $74^{\circ} 59,2^{\prime}$ & Canal San Blas \\
12 (Oeste) & 02 marzo de 2012 & $51^{\circ} 11,5^{\prime}$ & $74^{\circ} 42,4^{\prime}$ & Canal Guadalupe \\
\hline
\end{tabular}

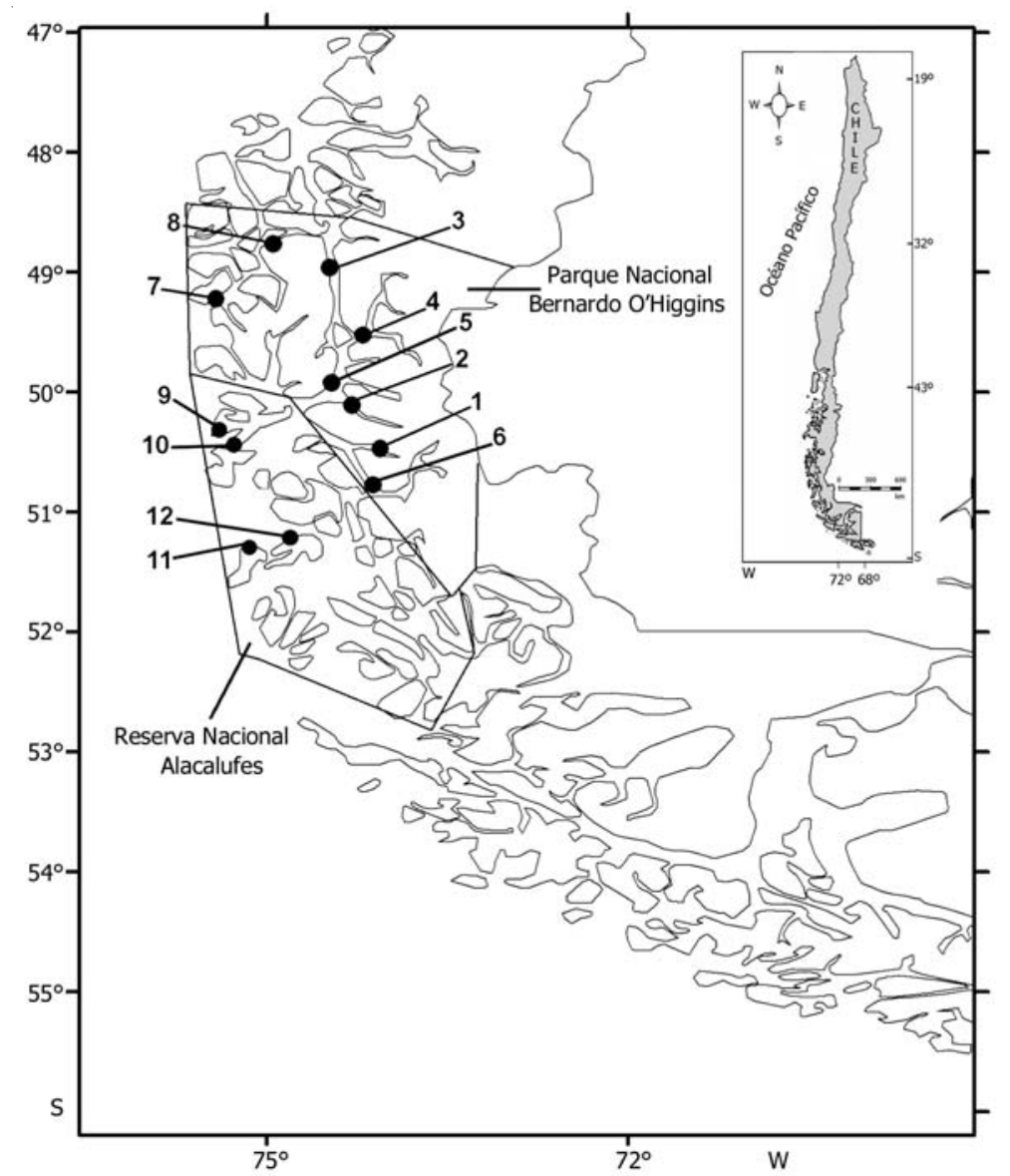

Figura 1. Sitios de estudio (círculos negros) con los números de cada estación, en los canales y fiordos subantárticos del sur de Chile / Study sites (black circles) with the numbers of each station, in the subAntarctic channels and fjords of southern Chile 
y $12 \mathrm{~cm}$ de malla externa, con períodos de reposo de $12 \mathrm{~h}$ (19:00-07:00 h), desplegada en el fondo entre los 0 y $15 \mathrm{~m}$ de profundidad. Además, se recolectaron a mano los peces que habitan las pozas intermareales de los sitios donde se instaló la red, abarcando un área aproximada de $120 \mathrm{~m}^{2}$. Las muestras biológicas fueron preservadas en una solución de alcohol al 90\% para su posterior identificación. Para la determinación taxonómica, se utilizó literatura especializada en grupos taxonómicos de peces del sur de Chile como Dyer (2000), Navarro \& Pequeño (1979), Lloris \& Rucabado (1991), Pequeño \& Lamilla (1995, 1996, 1997), Pequeño et al. (1995), Pequeño (1989, 1999, 2000) y Zama \& Cárdenas (1984).

\section{ANÁLISIS DE LOS DATOS}

Las medidas de diversidad se calcularon mediante estimadores no paramétricos, con el fin de evaluar si la riqueza observada de especies en los muestreos es representativa de la diversidad total de peces costeros de los canales analizados. Para lo cual, se utilizaron curvas de acumulación de especies generadas por el estimador de cobertura basado en la abundancia (ACE; Chao \& Lee 1992, Chao et al. 1993), mediante el programa EstimateS 8.2 (Colwell 2006). Además, a causa de los diferentes tamaños de muestreo entre los sitios, se utilizaron curvas de rarefacción para comparar la riqueza de especies entre ellos. Dado que el número de especies observadas en una muestra es en parte una función del número de individuos en una muestra, se calculó una estimación de la rarefacción de la riqueza de especies, es decir, el número medio esperado de las especies observadas mediante un número fijo de individuos (Gotelli \& Colwell 2001). Dos sitios exhibieron el menor número de ejemplares en el conjunto de datos, presentando 8 individuos. Por lo tanto, se realizó la rarefacción con este tamaño de muestra. Los datos fueron remuestreados 1.000 veces a través del método Montecarlo, para estimar la riqueza de especies, la diversidad alfa mediante el índice de Shannon (H') y la uniformidad de Hurlbert (PIE), utilizando el programa Ecosim 7.72 (Gotelli \& Entsminger 2008).

Por último, para evaluar las diferencias en la estimación de la riqueza de especies (ACE) y en la rarefacción estimada de la riqueza de especies, diversidad de Shannon y uniformidad de Hurlbert, entre los sectores Este y Oeste, se realizaron las pruebas estadísticas t de Student y en los casos donde no se cumplió el supuesto de igualdad de varianza, se utilizó la prueba de Wilcoxon (Zar 2010). Se consideraron significativas a un nivel de confianza del 95\% ( $P<0,05)$, utilizando para estos análisis el lenguaje $\mathrm{R}$ (R Development Core Team 2007).

\section{Análisis del ensamble de Peces}

Para docimar la hipótesis de si la estructura del ensamble de peces costeros varía en un gradiente longitudinal, se construyó un diseño de dos grupos, es decir, 6 estaciones de muestreo en el sector Oeste y 6 en el sector Este. Para identificar diferencias entre estos grupos, se utilizó el análisis de varianza no paramétrico mediante permutaciones (PERMANOVA). Por consiguiente, se utilizaron como variables 17 especies observadas en las estaciones de muestreo, cada variable fue transformada a raíz cuarta $\left(y^{\prime}=\sqrt{ } \sqrt{ } y^{\prime}\right)$, luego se calculó la distancia de disimilaridad de Bray-Curtis entre pares de estaciones, utilizando 4.999 permutaciones aleatorias sin restricciones. El análisis fue realizado por el programa computacional de permutaciones para análisis de varianza multivariado FORTRAN (Anderson 2005). Para visualizar los posibles cambios del ensamble de peces del gradientes Este-Oeste se utilizó la forma gráfica de escalamiento multidimensional MDS; para este método se utilizó la matriz de similitud de Bray-Curtis, donde los datos fueron trasformados a raíz cuarta, para reducir la contribución de las especies abundantes y equiparar a las especies raras (Clarke \& Warwick 2001), mediante el programa Primer 6 (Clarke \& Gorley 2006). Posteriormente, se realizó un análisis de porcentaje de disimilitud (SIMPER), para identificar aquellas especies que en promedio contribuyen en mayor medida a la diferencia entre grupos y similitud dentro de los grupos (Clarke 1993).

\section{Resultados}

\section{COMPOSICIÓN DE LA MUESTRA}

La captura total de peces fue de 380 individuos correspondientes a 12 familias y 17 especies (Tabla 2). Las especies Patagonotothen tessellata (Richardson, 1845) y Eleginops maclovinus (Cuvier, 1830), fueron aquellas que exhibieron la mayor frecuencia de ocurrencia, presentando ambas un $75 \%$, seguida por Patagonotothen longipes (Steindachner, 1876) con 50\%. La familia con mayor porcentaje de especies fue Notothenidae $(29,4 \%)$, seguida por Salmonidae (11,7\%).

\section{RIQUEZA Y DIVERSIDAD DE ESPECIES}

La estimación de la riqueza de especies mediante el estimador de cobertura basado en la abundancia (ACE) presentó diferencias significativas (prueba de Wilcoxon, $\mathrm{W}=32 ; P<0,05)$ entre las zonas analizadas. La riqueza de especies en las muestras de la zona Este (36 especies, 
estimación ACE) fue 4 veces mayor que las muestras de la zona Oeste (9 especies). Mientras que al agrupar ambas zonas, la curva de acumulación de especies alcanza un leve nivel asintótico de 32 especies (Fig. 2). No obstante, no se observaron diferencias entre los sectores Este y Oeste mediante el índice de diversidad Shannon (H') (prueba de t-Student $=0,63 ; P=0,54$ ). Este índice presentó el mayor valor en E1 de 1,67 y el menor en la E8 de 0,45; consecuentemente, el índice de uniformidad (PIE) no presentó diferencias entre los sectores (prueba de tStudent $=-0,16 ; P=0,87$ ) y exhibió el mayor valor 0,83 y el menor 0,29 en las estaciones 1 y 8, respectivamente (Tabla 3a). Las estimaciones de rarefacción (Tabla 3b), mostraron una riqueza esperada entre 4,5 (E1) y 2 (E4, E8, E12) especies, con límites de confianza del 95\% (LC95\%), de 3 a 6 especies para E1 y de 1 a 2 especies para E8. Los valores extremos de H' variaron entre 0,97 y 1,73 en E1 y entre 0,00 y 0,66 en E8, mientras que los máximos y mínimos de PIE se distribuyeron en E1 entre 0,64 y 0,93 y en E8 entre 0,00 y 0,54. Mediante las curvas de rarefacción, se aprecia una riqueza esperada de 9 especies para E6 y 6 para E1. Mientras que E3, el cual presentó el mayor número de individuos, exhibió una riqueza esperada de 4 especies (Fig. 3).

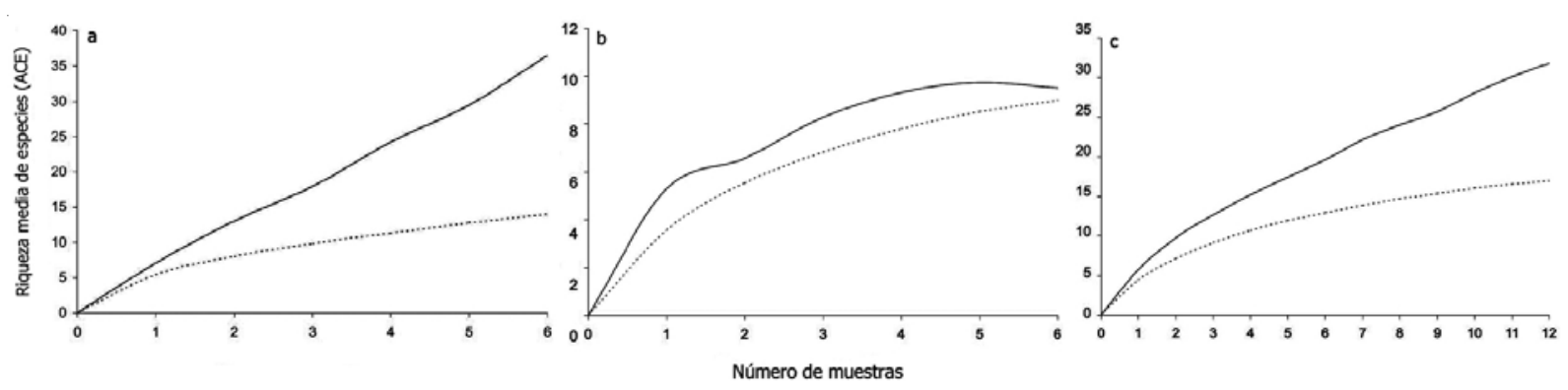

Figura 2. Curvas de acumulación de la riqueza media de especies a través del Estimador de Cobertura basado en la Abundancia (ACE) $(-)$ y la acumulación de especies observadas (--) para las estaciones del Este (a), Oeste (b) y el total de muestras (c) / Mean species richness accumulation curve using the Abundance-based Coverage Estimator (ACE) (-) and observed species accumulation (--) for the East (a) and West (b) stations and total samples (c)

Tabla 2. Abundancia por estaciones de muestreo (1 a 12) de las especies de peces, porcentaje de la frecuencia de ocurrencia (F) y número de individuos por especie $(\mathbf{N}) .\left(^{*}=\right.$ especies de categoría invasora) / Sampling stations (1 to 12 ) abundance of fish species, percentage of frequency of occurrence $(F)$ and number of individuals per species $(N) .{ }^{*}=$ invasive species category)

\begin{tabular}{|c|c|c|c|c|c|c|c|c|c|c|c|c|c|c|}
\hline Sitios de muestreo & 1 & 2 & 3 & 4 & 5 & 6 & 7 & 8 & 9 & 10 & 11 & 12 & $\mathrm{~N}$ & $\mathrm{~F}(\%)$ \\
\hline Odontesthes regia (Humboldt, 1821) & 1 & 4 & 51 & 0 & 0 & 0 & 0 & 0 & 0 & 0 & 0 & 0 & 56 & 25 \\
\hline Genypterus maculatus (Tschudi, 1846) & 0 & 0 & 0 & 0 & 0 & 1 & 0 & 0 & 0 & 0 & 0 & 0 & 1 & 8,3 \\
\hline Paranotothenia magellanica (Forster, 1801) & 5 & 1 & 0 & 0 & 0 & 0 & 1 & 0 & 0 & 0 & 1 & 0 & 8 & 33,3 \\
\hline Patagonotothen brevicauda (Lönnberg, 1905) & 0 & 0 & 0 & 0 & 0 & 0 & 0 & & 1 & 0 & 4 & 0 & 5 & 16,7 \\
\hline Patagonotothen cornucola (Richardson, 1844) & 0 & 0 & 0 & 0 & 0 & 0 & 5 & 0 & 7 & 0 & 8 & 5 & 25 & 33,3 \\
\hline Patagonotothen longipes (Steindachner, 1876) & 0 & 3 & 3 & 2 & 1 & 2 & 0 & 0 & 5 & 0 & 0 & 0 & 16 & 50 \\
\hline Patagonotothen tessellata (Richardson, 1845) & 8 & 6 & 0 & 0 & 2 & 2 & 8 & 3 & 0 & 3 & 1 & 4 & 37 & 75 \\
\hline Eleginops maclovinus (Cuvier, 1830) & 5 & 30 & 50 & 6 & 31 & 14 & 12 & 15 & 0 & 5 & 0 & 0 & 168 & 75 \\
\hline Champsocephalus esox (Günther, 1861) & 0 & 0 & 0 & 0 & 0 & 1 & 0 & 0 & 0 & 0 & 0 & 0 & 1 & 8,3 \\
\hline Cottoperca trigloides (Forster, 1801) & 0 & 0 & 0 & 0 & 0 & 1 & 0 & 0 & 0 & 0 & 1 & 0 & 2 & 16,7 \\
\hline Harpagifer bispinis (Schneider, 1801) & 4 & 0 & 0 & 0 & 0 & 0 & 0 & 0 & 0 & 0 & 17 & 0 & 21 & 16,7 \\
\hline Austrolycus depressiceps (Regan, 1913) & 0 & 0 & 0 & 0 & 0 & 0 & 15 & 0 & 4 & 0 & 0 & 0 & 19 & 16,7 \\
\hline Leptonotus blainvilleanus (Eydoux \& Gervais, 1837) & 0 & 0 & 0 & 0 & 0 & 1 & 0 & 0 & 0 & 0 & 0 & 0 & 1 & 8,3 \\
\hline Stromateus stellatus (Cuvier, 1829) & 0 & 0 & 0 & 0 & 1 & 0 & 0 & 0 & 0 & 0 & 0 & 0 & 1 & 8,3 \\
\hline Oncorhynchus kisutch* (Walbaum, 1792) & 0 & 0 & 0 & 0 & 0 & 1 & 0 & 0 & 0 & 0 & 0 & 0 & 1 & 8,3 \\
\hline Salmo salar* (Linnaeus, 1758$)$ & 4 & 0 & 7 & 0 & 3 & 3 & 0 & 0 & 0 & 0 & 0 & 0 & 17 & 33,3 \\
\hline Agonopsis chiloensis (Jenyns, 1842) & 0 & 1 & 0 & 0 & 0 & 0 & 0 & 0 & 0 & 0 & 0 & 0 & 1 & 8,3 \\
\hline Total individuos & 27 & 45 & 111 & 8 & 38 & 26 & 41 & 18 & 17 & 8 & 32 & 9 & 380 & \\
\hline
\end{tabular}




\section{Análisis del ensamble de Peces Este-Oeste}

La estructura del ensamble de peces presentó cambios significativos entre los grupos Este y Oeste (Permanova: $\mathrm{T}=2,04 ; P=0,01)$. Estas diferencias en el ensamble, también se pueden visualizar en el análisis de escalamiento multidimensional MDS (Fig. 4), con un valor del coeficiente de estrés de 0,14 . Dentro del grupo de estaciones asociadas al sistema de fiordos (categoría Este), la especie de mayor contribución al ensamble fue

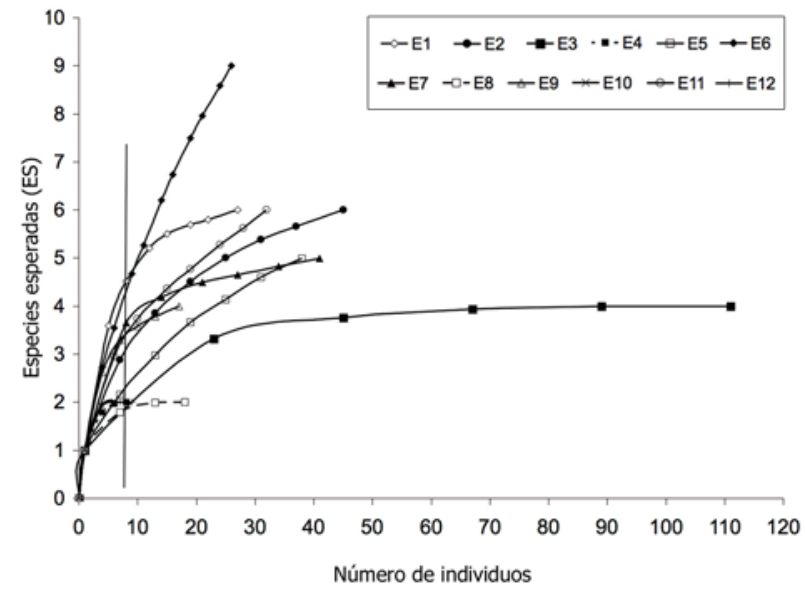

Figura 3. Curvas de rarefacción del número esperado de especies para las estaciones de muestreo. La barra indica los sub-muestreos para 8 especímenes / Rarefaction curves of the expected number of species for the sampling stations. The bar represents the subsample for 8 specimens
Eleginops maclovinus con un 47,8\%, mientras que dentro del grupo de estaciones con influencia al Océano Pacífico (categoría Oeste), fue Patagonotothen tessellata con un 46,5\%. Las especies que contribuyeron sobre el 50\% de disimilitud entre los ensambles Este y Oeste fueron $E$. maclovinus, Patagonotothen cornucola (Richardson, 1844), P. longipes, Salmo salar (Linnaeus, 1758) y Odontesthes regia (Humboldt, 1821) (Tabla 4).

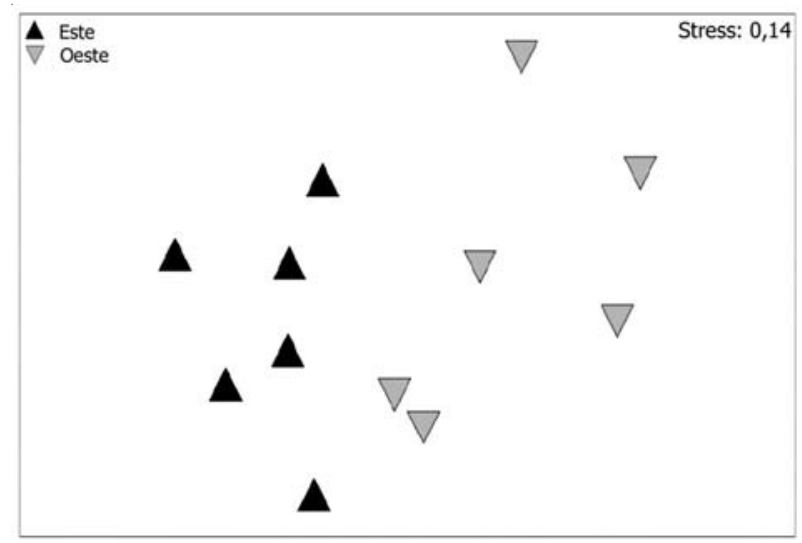

Figura 4. Escalamiento Multidimensional (MDS) $\quad$ (4999 permutaciones) para las estaciones de muestreo, los datos fueron transformados a raíz cuarta / Multi-dimensional Scaling (MDS) (4999 permutations) for the sampling stations, data were transformed to fourth root

Tabla 3. Riqueza, diversidad y uniformidad de peces costeros, estimadas mediante rarefacción para las estaciones de muestreo / Richness, diversity and uniformity of coastal fish, estimated by rarefaction for the sampling stations

\begin{tabular}{|c|c|c|c|c|c|c|}
\hline \multirow{2}{*}{$\begin{array}{l}\text { Estación } \\
\text { (E) }\end{array}$} & \multicolumn{3}{|c|}{$\begin{array}{l}\text { (a) Análisis utilizando } \\
\text { toda la matriz de datos }\end{array}$} & \multicolumn{3}{|c|}{$\begin{array}{l}\text { (b) Análisis utilizando } \\
\text { la rarefacción estimada }\end{array}$} \\
\hline & $\begin{array}{c}\text { Riqueza } \\
\text { media }\end{array}$ & $\begin{array}{l}\text { Diversidad } \\
\text { media }\end{array}$ & $\begin{array}{l}\text { Uniformidad } \\
\text { media }\end{array}$ & $\begin{array}{c}\text { Riqueza } \\
\text { media }\end{array}$ & Diversidad media & Uniformidad media \\
\hline 1 & 6 & 1,67 & 0,83 & $4,5(3-6)$ & $1,39(0,97-1,73)$ & $0,83(0,64-0,93)$ \\
\hline 2 & 6 & 1,10 & 0,54 & $3,1(2-5)$ & $0,84(0,38-1,38)$ & $0,54(0,00-0,87)$ \\
\hline 3 & 4 & 0,99 & 0,59 & $2,6(2-4)$ & $0,79(0,38-1,21)$ & $0,59(0,25-0,75)$ \\
\hline 4 & 2 & 0,56 & 0,43 & $2 \quad(2-2)$ & $0,56(0,56-0,56)$ & $0,43(0,43-0,43)$ \\
\hline 5 & 5 & 0,71 & 0,33 & $2,3(1-4)$ & $0,49(0,00-1,07)$ & $0,33(0,00-0,64)$ \\
\hline 6 & 9 & 1,60 & 0,70 & $4,3(2-6)$ & $1,19(0,38-1,73)$ & $0,71(0,25-0,93)$ \\
\hline 7 & 5 & 1,39 & 0,74 & $3,7(2-5)$ & $1,17(0,69-1,49)$ & $0,74(0,54-0,86)$ \\
\hline 8 & 2 & 0,45 & 0,29 & $2,0(1-2)$ & $0,41(0,00-0,66)$ & $0,29(0,00-0,54)$ \\
\hline 9 & 4 & 1,23 & 0,73 & $3,4(2-4)$ & $1,11(0,69-1,32)$ & $0,73(0,54-0,82)$ \\
\hline 10 & 6 & 0,66 & 0,54 & $3,3(2-5)$ & $0,66(0,66-0,66)$ & $0,54(0,54-0,54)$ \\
\hline 11 & 6 & 1,28 & 0,66 & $3,4(2-5)$ & $1,02(0,38-1,49)$ & $0,65(0,25-0,86)$ \\
\hline 12 & 2 & 0,69 & 0,56 & $2,0(2-2)$ & $0,68(0,66-0,69)$ & $0,55(0,54-0,57)$ \\
\hline
\end{tabular}


Tabla 4. Análisis del porcentaje de contribución SIMPER muestra las especies que más contribuyen a la disimilitud entre los ensambles de peces costeros de las estaciones de muestreo Este y Oeste. Abundancia media (Ab. Media) (*: especies de categoría invasora) / SIMPER percentage contribution analysis shows the species that contribute most to the dissimilarity between the coastal fish assemblages of the sampling stations East and West. Mean abundance (Ab. Media) (*: invasive species category)

\begin{tabular}{lccc}
\hline Especies & $\begin{array}{c}\text { Este } \\
\text { Ab. Media }\end{array}$ & $\begin{array}{c}\text { Oeste } \\
\text { Ab. Media }\end{array}$ & \% contribución \\
\hline Eleginops maclovinus & 22,67 & 5,33 & 14,76 \\
Patagonotothen cornucola & 0 & 4,17 & 11,75 \\
Patagonotothen longipes & 1,83 & 0,83 & 11,56 \\
Salmo salar* & 2,83 & 0 & 10,29 \\
Odontesthes regia & 9,33 & 0 & 9,23 \\
Patagonotothen tessellata & 3 & 3,17 & 9,02 \\
Austrolycus depressiceps & 0 & 3,17 & 5,79 \\
Paranotothenia magellanica & 1 & 0,33 & 5,71 \\
Promedio de disimilitud & 69,7 & & \\
\hline
\end{tabular}

\section{Discusión}

\section{VARIACIÓN EN LA DIVERSIDAD DE ESPECIES}

Se observaron diferencias espaciales en la riqueza de peces costeros en los canales y fiordos subantárticos de la zona central de la Patagonia. La mayor riqueza estimada se presentó en las estaciones del sector Este (36 especies), mientras que el sector Oeste obtuvo 9 especies estimadas. Esto podría ser consecuencia principalmente del elevado número de individuos capturados en las estaciones del sector Este. Por ejemplo, Odontesthes regia, especie de hábitos gregarios que frecuenta los ambiente estuarinos para desovar (Oliver-Schneider 1943), presentó un elevado número de ejemplares en la estación 3. Otro factor que influiría en la estimación de la riqueza, tiene relación al alto número de especies raras o poco frecuentes, como por ejemplo, Leptonotus blainvilleanus (Eydoux \& Gervais 1837), especie asociada a Ulva sp. (M. Hüne, obs. pers.), alga frecuente en ambientes de baja salinidad (Soto et al. 2012) dentro del sistema de canales del sector Este. Por otro lado, no se observaron diferencias significativas en la diversidad entre ambos sectores. Esto principalmente, debido a la ocurrencia y abundancia de especies como Patagonotothen cornucola y Austrolycus depressiceps (Regan, 1913), presentes sólo en el sector Oeste.

La presencia de una mayor riqueza de peces en el sector Este, sistema de fiordos y canales asociados a fuentes de aporte glacial, se contrapone a los resultados obtenidos para invertebrados bentónicos de la zona central de la
Patagonia (Thatje \& Mutschke 1999, Häussermann 2006), e.g., Häussermann (2006) propone una disminución de la diversidad de anémonas en el intermareal y aguas someras (0-15 m) de los fiordos interiores de la zona norte de la Patagonia, a causa de una combinación de diversos factores, como la baja salinidad y amplitud de marea que afectan a las larvas de estas especies. Estos autores señalan que el estrés causado por la sedimentación glacial, influiría en la baja diversidad de invertebrados en los sectores asociados a ventisqueros y canales interiores de la zona central de la Patagonia (Thatje \& Mutschke 1999, Ríos et al. 2005, Häussermann 2006). Esta diferencia se podría atribuir a la capacidad de movilidad que presentan los peces. Además, se ha reportado en peces subantárticos como E. maclovinus, un incremento en la actividad natatoria con el aumento de la temperatura durante el verano-otoño (época del muestreo del presente estudio) (Fernández et al. 2002, Vanella \& Calvo 2005). Por lo tanto, la movilidad sumada a la mayor actividad natatoria, podría influir en la distribución de los peces en ambientes con mayor descarga de sedimentos, donde los invertebrados bentónicos sésiles no logran asentarse.

Por otro lado, estudios realizados en zonas con influencia oceánica caracterizaron biotopos semiexpuestos, con salinidades $>32$ y la presencia de Durvillaea antarctica (Chamisso) Hariot, 1892 y Macrocystis pyrifera (Linnaeus) Agardh, 1820 (Soto et al. 2012) junto con el reporte de un total de 11 especies de 
peces para el sector Oeste, aunque el máximo por estación no superó las 5 especies (Sielfeld et al. 2006). Estos resultados no se alejan de los obtenidos por nuestro estudio (9 especies) para la misma área y la diferencia se podría atribuir a las diferentes metodologías de muestreo utilizadas (red de trasmallo y colecta manual en el presente trabajo y espineles de fondo y colecta manual en Sielfeld et al. 2006). Esta diferencia podría explicar la ausencia de especies como por ejemplo, Sebastes oculatus (Valenciennes, 1833) y Salilota australis (Günther, 1878), características de profundidades superiores al rango batimétrico estudiado (Lloris \& Rucabado 1991). Cabe destacar, que en zonas intermareales expuestas directamente al oleaje oceánico se han reportado especies como Gobiesox marmoratus (Jenyns, 1842) y Sicyaces sanguineus (Müller \& Troschel, 1843) (Venegas \& Sielfeld 1998), especies que poseen una estructura funcional (ventosa ventral) para adherirse a las rocas intermareales, debido a que habitan en zonas con alta exposición al oleaje (Vera \& Pequeño 2001), esto probablemente difiere de los ambientes semi-expuestos observados en el presente estudio.

\section{ESTRUCTURA DE LOS ENSAMBLES}

En el análisis espacial fueron identificados 2 grupos correspondientes al sector Este y Oeste (Fig. 4), los que presentaron diferencias significativas. Este patrón longitudinal, igual que en comunidades de macroinvertebrados bentónicos del área de estudio (Montiel et al. 2011¹), podría estar influida por el aporte de aguas dulces y sedimentos originados por el Campo de Hielo Sur, que causa variaciones longitudinales en las condiciones oceanográficas del área (e.g., temperatura y salinidad), presentando un gradiente estuarino-marino. Estas variables ambientales, podrían explicar en este estudio la elevada contribución en la disimilitud entre los grupos de especies como el róbalo (Eleginops maclovinus), que se asocian normalmente a zonas estuarinas con baja salinidad (Pequeño 1979). Cabe destacar, la presencia del salmón del Atlántico (Salmo salar) y el salmón Coho (Oncorhynchus kisutch Walbaum, 1792). Estas especies fueron introducidas en la región de Magallanes entre la década de 1920 y 1980, respectivamente (Basulto 2003). El impacto de estas especies invasoras en la depredación y competencia con las especies nativas, podría influir en la composición de los ensambles de peces costeros (Fernandez et al. 2000, Soto et al. 2001, Buschmann et al. 2006).

En conclusión, los cambios espaciales observados en el área de estudio en relación a la riqueza de especies, fue mayor en las localidades del sector Este del sistema de fiordos y canales. También se sugiere un patrón de estructuración definido de las especies en cuanto a la composición. Sin embargo, la ausencia de datos distribuidos estacionalmente a causa de la lejanía y difícil acceso a las zonas muestreadas, no permite una comparación de la variación temporal en la diversidad de especies y composición del ensamble. En este contexto, se requiere mayor información sobre el efecto estacional de las variables oceanográficas y cómo estos procesos influyen en las características de los ensambles y comunidades. Una mejor comprensión de estas interacciones, permitirá el diseño de planes de conservación que evalúen el impacto del cambio climático y de actividades humanas en los canales y fiordos subantárticos.

\section{Agradecimientos}

Al apoyo logístico brindado por el proyecto 'Caracterización Territorial del Parque Nacional Bernardo O’Higgins, su Potencial Económico, Turístico, Científico y Cultural' (INNOVA-CORFO 08CTU01-20), desarrollado por la Fundación CEQUA y la Corporación Nacional Forestal de Chile (CONAF). A la Dra. Verena Häussermann por el apoyo logístico brindado para la toma de muestras en las estaciones 9 a la 12. Al Dr. Cristian Aldea por los aportes realizados a este escrito. M. Hüne agradece a la beca de Magíster del Programa de Financiamiento BASAL código PFB-23-2008 otorgado por el Instituto de Ecología y Biodiversidad (IEB). J. Ojeda agradece al programa de Magíster en Ciencias con Mención en Manejo y Conservación de Recursos Subantárticos de la Universidad de Magallanes y a la beca de Magíster del Proyecto ICM, código P05-002 otorgada por el IEB.

\section{LITERATURA CITADA}

Anderson MJ. 2005. PERMANOVA: a FORTRAN computer program for permutational multivariate analysis of variance, 24 pp. Department of Statistics, University of Auckland, Auckland. < http://www.stat.auckland.ac.nz/ mja/prog/ PERMANOVA_UserNotes.pdf>

\footnotetext{
${ }^{1}$ Montiel A, C Aldea \& T Césped. 2011. Patrones de distribución espacial en comunidades macrobentónicas de fiordos y canales chilenos: relación entre abundancia y biomasa. XIV Congreso Latino Americano de Ciencias del Mar - XIV COLACMAR. Camboriú, Brasil. 4 pp.
} 
Andrade S. 1991. Geomorfología costera y antecedentes oceanográficos físicos de la región de Magallanes, Chile (48$56^{\circ}$ S). Anales del Instituto de la Patagonia 20(1): 135-151.

Basulto S. 2003. El largo viaje de los salmones: una crónica olvidada, propagación y cultivo de especies acuáticas en Chile, 299 pp. Editorial Maval, Santiago de Chile.

Buschmann A, V Riquelme, M Hernández-González, D Varela, J Jiménez, L Henríquez, P Vergara, R Guíñez \& L Filún. 2006. A review of the impacts of salmonid farming on marine coastal ecosystems in the southeast Pacific. ICES Journal of Marine Science 63: 1338-1345.

Bustos CA, MF Landaeta \& F Balbontín. 2011. Ichthyoplankton spatial distribution and its relation with water column stratification in fjords of southern Chile (46 $48^{\prime}-50^{\circ} 09^{\prime} S$ ) in austral spring 1996 and 2008. Continental Shelf Research 31: 293-303.

Chao A \& S Lee. 1992. Estimating the number of classes via sample coverage. Journal of the American Statistical Association 87: 210-217.

Chao A, M-C Ma \& MCK Yang. 1993. Stopping rules and estimation for recapture debugging with unequal failure rates. Biometrika 80: 193-201.

Clarke KR. 1993. Non-parametric multivariate analyses of changes in community structure. Australian Journal of Ecology 18: 117-143.

Clarke KR \& R Warwick. 2001. Change in marine communities: an approach to statistical analysis and interpretation, 168 pp. Natural Environment Research Council, Plymouth Marine Laboratory, Plymouth.

Clarke KR \& RN Gorley. 2006. PRIMER v6: User Manual/ Tutorial, 190 pp. PRIMER-E, Plymouth.

Colwell R. 2006. EstimateS: statistical estimation of species richness and shared species from samples. Versión 8.2. [on line] <http://viceroy.eeb.uconn.edu/EstimateS>

Dyer B. 2000. Revisión sistemática de los pejerreyes de Chile (Teleostei, Atheriniformes). Estudios Oceanológicos 19: 99127.

Fernández M, E Jaramillo, PA Marquet, CA Moreno, SA Navarrete, FP Ojeda, CR Valdovinos \& JA Vásquez. 2000. Diversity, dynamics and biogeography of Chilean benthic nearshore ecosystems: an overview and guidelines for conservation. Revista Chilena de Historia Natural 73: 797-830.

Fernández DA, J Calvo, M Wakeling, FA Vanella \& IA Johnston. 2002. Escape performance in the sub-Antarctic notothenioid fish Eleginops maclovinus. Polar Biology 25: 914-920.

González-Wevar C, M Hüne, JI Cañete, A Mansilla, T Nakano \& E Poulin. 2012. Towards a model of postglacial biogeography in shallow marine species along the Patagonian Province: lessons from the limpet Nacella magellanica (Gmelin, 1791) BMC Evolutionary Biology 12: 139. <doi:10.1186/1471-2148-12-139>
Gotelli N \& R Colwell. 2001. Quantifying biodiversity: procedures and pitfalls in the measurement and comparison of species richness. Ecology Letters 4: 379-391.

Gotelli N \& G Entsminger. 2008. EcoSim: null models software for ecology. Versión 7.72 Acquired Intelligence \& Kesey-Bear. Jericho, VT 05465. [on line] <http:// www.garyentsminger.com/ecosim.htm>

Häussermann V. 2006. Biodiversity of Chilean sea anemones (Cnidaria: Anthozoa): distribution patterns and zoogeographic implications, including new records for the fjord region. Investigaciones Marinas 34(2): 23-35.

Hulton N, R Purves, R McCulloch, De Sugden \& M Bentley. 2002. The Last Glacial Maximum and deglaciation in southern South America. Quaternary Science Reviews 21: 233-241.

Kvalseth T. 1991. Note on biological diversity, evenness, and homogeneity measures. Oikos 62: 123-127.

Landaeta MF, CA Bustos, P Palacios-Fuentes, P Rojas \& F Balbontín. 2011. Distribución del ictioplancton en la Patagonia austral de Chile: potenciales efectos del deshielo de Campos de Hielo Sur. Latin American Journal of Aquatic Research 39(2): 236-249.

Lloris D \& J Rucabado. 1991. Ictiofauna del Canal Beagle (Tierra del Fuego), aspectos ecológicos y análisis biogeográfico. Instituto Español de Oceanografía, Publicaciones Especiales 8: 1-182.

Navarro J \& G Pequeño. 1979. Peces litorales de los archipiélagos de Chiloé y Los Chonos, Chile. Revista de Biología Marina y Oceanografía 16(3): 255-309.

Oliver-Schneider C. 1943. Catálogo de los peces marinos del litoral de Concepción y Arauco. Boletín de la Sociedad de Biología de Concepción 17: 75-126.

Pequeño G. 1979. Antecedentes alimentarios de Eleginops maclovinus (Valenciennes, 1830) (Teleostomi Nototheniidae), en Mehuín, Chile. Acta Zoológica Lilloana 35(1): 207-230.

Pequeño G. 1989. Peces de Chile. Lista Sistemática Revisada y Comentada. Revista de Biología Marina 24(2): 1-132.

Pequeño G. 1999. Peces del crucero CIMAR-Fiordo 2, a los canales patagónicos de Chile, con consideraciones ictiogeográficas. Ciencia y Tecnología del Mar 22: 165-179.

Pequeño G. 2000. Peces del crucero CIMAR-Fiordo 3, a los canales del sur de Magallanes (ca. 55 S), Chile. Ciencia y Tecnología del Mar 23: 83-94.

Pequeño G \& J Lamilla. 1995. Peces intermareales de la costa de Llanquihue (Chile): composición taxonómica, abundancia relativa y gradiente de distribución longitudinal. Revista de Biología Marina 30(1): 7-27.

Pequeño G \& J Lamilla. 1996. Preliminary report on the demersal fish material collected during the 'Victor Hensen' Magellan Campaign. Berichte zur Polarforschung 190: 68-70. 
Pequeño G \& J Lamilla. 1997. Peces litorales del Crucero CIMAR 1 Fiordos. Ciencia y Tecnología del Mar 20: 165171.

Pequeño G, J Lamilla, D Lloris \& J Rucabado. 1995. Comparación entre las ictiofaunas intermareales de los extremos austral y boreal de los canales patagónicos. Revista de Biología Marina 30(2): 155-177.

Pickard GL. 1971. Some physical oceanographic features of inlets of Chile. Journal of the Fisheries Research Board of Canada 28: 1077-1106.

Pickard GL. 1973. Water structure in Chilean fjords. In: Fraser R (ed). Oceanography of the South Pacific, pp. 95-104. New Zealand National Commission for UNESCO, Wellington.

Pinochet P \& S Salinas. 1996. Estructura térmica y salina de fiordos y canales adyacentes a Campos de Hielo Sur. Ciencia y Tecnología del Mar 19: 93-122.

Porter C \& A Santana. 2003. Rápido retroceso, en el siglo 20, del ventisquero Marinelli en el campo de hielo de la Cordillera Darwin. Anales del Instituto de la Patagonia 31: 17-26.

R Development Core Team. 2007. R: A language and environment for statistical computing. R Foundation for Statistical Computing, Vienna. [on line] <http://www.rproject.org>

Ricotta C. 2003. On parametric evenness measures. Journal of Theorical Biology 222: 189-197.

Rignot E, A Rivera \& G Casassa. 2003. Contribution of the Patagonia Icefields of South America to Sea Level Rise. Science 302: 434-437.

Ríos C, E Mutschke, A Montiel, D Gerdes \& W Arntz. 2005. Soft-bottom macrobenthic faunal associations in the southern Chilean glacial fjord complex. Scientia Marina 69(2): 225-236.

Sielfeld W, G Guzman \& N Amado. 2006. Distribución de peces del litoral rocoso de los canales patagónicos

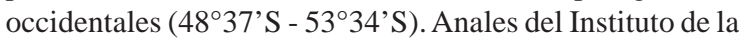
Patagonia 34: 21-32

Sievers H \& N Silva. 2006. Masas de agua y circulación en los canales y fiordos australes. En: Silva N \& S Palma (eds). Avances en el conocimiento de las aguas interiores chilenas, Puerto Montt a cabo de Hornos, pp. 53-58. CONA, Valparaíso.
Silva N \& C Calvete. 2002. Características oceanográficas físicas y químicas de canales australes chilenos entre golfo de Penas y el estrecho de Magallanes (Crucero CIMARFiordo 2. Ciencia y Tecnología del Mar 25(1): 23-88.

Soto D, F Jara \& C Moreno. 2001. Escape salmon in the inner seas, southern Chile: facing ecological and social conflicts. Ecological Applications 11(6): 1750-1762.

Soto E, P Báez, ME Ramírez, S Letelier, J Naretto \& A Rebolledo. 2012. Biotopos marinos intermareales entre Canal Trinidad y Canal Smyth, Sur de Chile. Revista de Biología Marina y Oceanografía 47(2): 177-191.

Sudgen E, J Hulton \& S Purves. 2002. Modelling the inception of the Patagonia icesheet. Quaternary International 95-96: 55-64.

Thatje S \& E Mutschke. 1999. Distribution of abundance, biomass, production and productivity of macrozoobenthos in the sub-Antarctic Magellan Province (South America). Polar Biology 22: 31-37.

Vanella FA \& J Calvo. 2005. Influence of temperature, habitat and body mass on routine metabolic rates of subantarctic teleosts. Scientia Marina 69(2): 317-323.

Venegas C \& W Sielfeld. 1998. Catálogo de los vertebrados de la Región de Magallanes y Antártica Chilena, 122 pp. Ediciones Universidad de Magallanes, Punta Arenas.

Vera R \& G Pequeño. 2001. Comparación de caracteres merísticos y morfométricos entre peces del género Sicyases del archipiélago de Juan Fernández, Valparaíso y Valdivia (Osteichthyes: Gobiesocidae). Investigaciones Marinas 29(2): 3-14

Worm B, M Sandow, A Oschlies, H Lotze \& R Myers. 2005. Global patterns of predator diversity in the open oceans. Science 309: 1365-1369.

Zama A \& E Cárdenas. 1984. Descriptive catalogue of marine and freshwater fishes from the Aysén Region, southern Chile, with zoogeographical notes on the fish fauna. Introduction into Aysén Chile of Pacific Salmon, Servicio Nacional de Pesca / Japan International Cooperation Agency 9: 1-75.

Zar J. 2010. Bioestatistical analysis, 947 pp. Prentice-Hall, Upper Saddle River.

Recibido el 29 de junio de 2012 y aceptado el 3 de octubre de 2012

Editor Asociado: Mauricio Landaeta D. 